\title{
MEJORANDO LA COMPETITIVIDAD DE LAS MICROEMPRESAS EN LA REGIÓN BRUNCA DE COSTA RICA ${ }^{1}$
}

\section{IMPROVISING THE COMPETITIVENESS OF MICROENTERPRISES IN THE BRUNCA REGION OF COSTA RICA}

\author{
Manuel Chaves Núñez \\ Andrey Valenciano Salazar** \\ Grethel Vega Arce*** \\ Gabriela Ortiz Valverde ${ }^{* * * *}$
}

RESUMEN

Se descubren limitaciones en los conocimientos empresariales con que cuentan los dueños(as) de las microempresas en la Región Brunca de Costa Rica, mediante un diagnóstico de las capacidades competitivas de estas; sin embargo mediante un proceso combinado de capacitación y seguimiento se puede revertir la situación a la que se enfrentan dichos microempresarios(as), generando mayores habilidades, las cuales les permitan competir en un sistema económico cada vez más demandante.

PALABRAS CLAVE: COSTA RICA * PEQUEÑAS EMPRESAS * FORMACIÓN PROFESIONAL * COMPETITIVIDAD * BIENESTAR SOCIAL * POBREZA

1 Los autores son investigadores del proyecto FOCCO- Mipymes de la Escuela de Economía de la Universidad Nacional (UNA) de Costa Rica. El objeto de estudio son las micro, pequeñas y medianas empresas de Costa Rica, y sus ejes de acción principales son la investigación de sectores productivos, capacitación y asesoría empresarial.

* Escuela de Economía de la Universidad Nacional de Costa Rica mcha@una.ac.cr

** Escuela de Economía de la Universidad Nacional de Costa Rica jvalenci@una.ac.cr

*** Escuela de Economía de la Universidad Nacional de Costa Rica grethelva@hotmail.com

**** Escuela de Economía de la Universidad Nacional de Costa Rica hecsoime@gmail.com 


\title{
ABSTRACT
}

\begin{abstract}
Through a diagnostic of competitive capabilities of small companies in the Brunca Region of Costa Rica, we discover limitations in the knowledge that business owners have. However, a combined process of training and monitoring can achieve to reverse the situation faced by these entrepreneurs and improve aptitudes that allow them to compete in an economic system increasingly demanding.
\end{abstract}

KEYWORDS: COSTA RICA * SMALL ENTERPRISES * VOCATIONAL TRAINING * COMPETITIVENESS * SOCIAL WELFARE * POVERTY

\section{INTRODUCCIÓN}

El bienestar socioeconómico de las personas depende en gran parte de las oportunidades que estas tengan dentro de una sociedad o sistema económico para generar ingresos y realizar un nivel de consumo que les permita solventar sus necesidades. La generación de emprendimientos productivos y creación de micro, pequeñas y medianas empresas (MiPyMEs) se ha constituido en Costa Rica en una alternativa para solventar las condiciones de pobreza $y$ vulnerabilidad social de las familias. Sin embargo, el aporte de estas unidades productivas al mejoramiento de las condiciones de vida de las personas está limitado por la baja capacidad de competir en el mercado local e internacional que estas muestran.

Las MiPyMEs presentan un bajo nivel de productividad debido a una serie de limitantes como: falta de financiamiento, limitada capacidad gerencial, carencia de una visión de largo plazo, carencia de estructuración de costos, escasez de procesos de innovación, entre otros. Estos factores surgen en gran medida por el bajo nivel de educación y formación técnica y empresarial de los(as) responsables de estas unidades productivas. Por tanto, la formación empresarial continua es un instrumento adecuado para mejorar las capacidades competitivas de los trabajadores(as) y administradores(as) de estas empresas y el bienestar socioeconómico de las personas.

Considerando el contexto social y económico de las MiPyMEs descrito, el presente artículo pretende responder la siguiente interrogante: ¿Cómo desarrollar una estrategia de intervención en la formación empresarial que permita mejorar las capacidades competitivas de las empresas y las condiciones socioeconómicas de las personas en condiciones de pobreza $y$ vulnerabilidad social? La experiencia desarrollada en la Región Brunca del país por parte del proyecto "Formación Continua de las Capacidades Competitivas de las Micro, Pequeñas y Medianas Empresas de Costa Rica" (FOCCO-MIPYME) de la Escuela de Economía de la Universidad Nacional de Costa Rica, se ha basado en una estrategia de formación continua que brinda una solución bastante satisfactoria a la interrogante y que ha rendido resultados positivos.

El presente artículo consta de cinco apartados: el primero incluye la introducción. El segundo, caracteriza el comportamiento de las MiPyMEs en la economía costarricense, el aporte que realizan a las familias en condiciones de pobreza y vulnerabilidad social, así como, los factores que explican su baja capacidad competitiva. El tercer apartado describe el método de formación continua en empresariedad utilizado por el proyecto FOCCO-MIPYME. El cuarto, narra los principales resultados de la intervención en los(as) beneficiarios(as) del proyecto y el estudio de casos exitosos de empresariedad. Finalmente, el quinto segmento propone lineamientos generales de estrategias de extensión, empresariedad y política pública para el mejoramiento de las capacidades competitivas de las MiPyMEs y el efecto de estas en el bienestar socioeconómico de las personas.

\section{CARACTERIZACIÓN DE LAS MIPYMES}

Según el decreto del Presidente de la República de Costa Rica y del Ministerio de Economía, Industria y Comercio nro. 264226: 
... las micro, pequeñas y medianas empresas conocidas como Pymes puede ser definidas a partir del número de empleados que poseen. Por lo tanto, una micro empresa será aquella que tiene entre 1 y 5 empleados, una pequeña empresa es aquella que cuenta con 6 $y$ hasta 30 empleados $y$ una mediana empresa tiene entre 31 y 100 trabajadores. Sin duda alguna, este criterio puede fortalecerse considerando variables adicionales como el nivel de inversión, ventas, maquinaria y equipo, dotación de infraestructura, entre otros.

Para definir el tamaño de las PyMEs a partir del criterio anterior no resulta del todo válido, de ahí que sugieren incorporar argumentos relacionados con eficiencia técnica e institucional, donde es relevante la relación entre eficiencia y costos de transacción, la competencia imperfecta $y$ el ciclo de vida de la empresa (Tommaso y Dubbini, 2000: 9. Citado por Gómez, 2002: 129).

En el caso de las microempresas atendidas por el proyecto FocCo-MIPYME, los diagnósticos de empresariedad y perfil socioeconómicos realizados de forma previa a los procesos de formación empresarial, han evidenciado una serie de características de dichas unidades productivas. Un estudio realizado con 171 microempresarios(as) indicó que el principal motivo para crear la empresa es poder complementar los ingresos del hogar, este fue el único motivo para $37,4 \%$ de las personas consultadas y uno de los criterios utilizados en combinación con otros criterios para un 36,6\% de los(as) empresarios(as). Cabe destacar que solo 3,5\% de los(as) entrevistados(as) indicaron como motivo para crear la empresa, el no haber encontrado trabajo como asalariado.

Sin embargo, las condiciones en que las MiPyMEs se desenvuelven en la región no les son particularmente favorables. La causa de esta situación es la:

... insuficiencia de competencias endógenas, tales como el bajo grado de adopción tecnológica, la poca calificación de sus trabajadores(as) y/o del propio(a) empresario(a), alta fragilidad administrativa $y$ baja productividad, pero también es real que ciertas condiciones del entorno e institucionales las afectan en mayor grado que a las grandes empresas (Zevallos, 2003: 58).

Estos mismos problemas han sido evidenciados por el proyecto a través de los perfiles socioeconómicos y de empresariedad que se han realizado. En cuanto a ingresos que perciben los hogares, un estudio de empresariedad aplicado a un grupo de 156 microempresarios(as), 38 hombres y 118 mujeres, atendidos por el proyecto en la Región Brunca de Costa Rica para los años 2006 y 2007, mostró que el ingreso promedio del hogar era de $\$ 115000,00$ y que el $50 \%$ cuenta con menos de \& 75000,00 . Similares resultados se han obtenido en los perfiles socioeconómicos de las personas atendidas durante el 2008, los cuales muestran una entrada de dinero media de $\$ 106300,00$, mientras que el 50\% de la muestra percibe menos de $\$ 80000,00$.

Los niveles de educación de los dueños(as) de estas microempresas son muy bajos. De acuerdo con los estudios mencionados, la mayoría de las personas no han concluido la secundaria $(87,7 \%$ en $2006-2007$ y $79,8 \%$ en 2008 ), en varios casos no poseen ningún tipo de educación (6\% en 2006-2007 y 2,3\% en 2008) y un alto porcentaje no ha concluido la primaria (15,1\% en $2006-2007$ y 17,2\% en 2008).

La mayoría de las empresas $(73,6 \%)$ con las que el proyecto ha trabajado, son lideradas principalmente por mujeres con bajos niveles de educación y se han formado para complementar los bajos ingresos de los hogares. Además, el $85 \%$ de ellas no poseen todos los trámites de formalización.

A pesar del fin con que fueron creadas estas unidades productivas, el aporte que realizan a los ingresos del hogar ha sido muy limitado. El 50\% de los(as) microempresarios(as) perciben menos de $\$ 20000,00$ de entradas de dinero de la empresa y solamente, el $13,1 \%$ percibe más de $\$ 100000,00$.

Este bajo aporte a los ingresos del hogar está condicionado por varios factores críticos 
que limitan la competitividad de estas empresas. Por el lado de la oferta, que se refiere a los recursos con que cuenta la producción y la organización, se enfrentan a una serie de problemas, dentro de los que se pueden citar: falta de financiamiento, procesos productivos intensivos en mano de obra, limitada capacidad gerencial, carencia de una visión de largo plazo, estructuración de costos ineficiente, poca innovación $y$ diseño de productos. Por el lado de la demanda, los elementos más perjudiciales son: limitado uso del mercadeo y la publicidad, desconocimiento de canales de comercialización, inadecuada fijación de precios, carencia de nichos de mercado identificados y desconocimiento de gustos y preferencias de los consumidores.

Estos problemas son reflejados dentro de los datos obtenidos en los perfiles de empresariedad aplicados a los(as) microempresarios(as) en el año 2007-2008, en donde 3 de cada 10 no registraban de ninguna forma sus costos de producción y el restante $70 \%$ de los(as) productores(as) aplicaban técnicas propias para registrar los costos.

Otra variable que muestra la falta de capacitación que tienen los(as) empresarios(as) dueños(as) de microempresas en Costa Rica es que el $23,7 \%$ de la muestra no tenía ningún conocimiento en gestión estratégica y en un $27 \%$ era muy escaso. Otras variables que destacan son la falta de procesos y transferencia de información dentro de la empresa para controlar la calidad del producto, en donde la mitad de los(as) emprendedores(as) consultados(as) dijeron no aplicar ninguna técnica para el control de calidad.

En cuanto al registro de ventas, el 24,2\% de los(as) dueños(as) nunca las registraban y el $28,2 \%$ solamente a veces lo hace. En el caso de las compras, la situación no varía significativamente, ya que un $22 \%$ afirman nunca registrar las compras de insumos y el 30,1\% solamente a veces hacían el registro.

En cuanto a la comercialización, un $22 \%$ de los(as) microempresarios(as) dijeron no saber nada de mecanismos de comercialización y $56 \%$ conocían poco de estos. Además, 4 de cada 10 microempresarios(as) no tenían ningún registro de clientes, mientras que 2 de cada 10 solamente algunas veces lo hacían.

Otro aspecto que muestra la falta de habilidades de los pequeños empresarios de la región Brunca del país, es el escaso conocimiento de nuevas tecnologías de la información, ejemplo de ello es que el $80 \%$ de los(as) emprendedores(as) no tenían ningún dominio de internet.

Estos datos son un reflejo de la falta de capacidades que tienen los(as) microempresarios (as) en la Región Brunca del país, con lo cual se justifica la intervención y capacitación de estos por medio de un proyecto que les provea de herramientas para operar de forma más adecuada su empresa $y$ así reducir el porcentaje de empresas que deben cerrar o retirarse de la industria por no tener la capacidad de enfrentar la competencia.

Un aspecto relevante es que los(as) mismos(as) microempresarios(as) están conscientes de la necesidad de aplicar nuevas técnicas en el manejo de sus emprendimientos productivos, por ejemplo: $92,3 \%$ consideran importante o muy importante el desarrollo de estrategias de mercado.

Uno de los factores más limitantes para las microempresas es la falta de financiamiento, sin embargo, muchas veces las entidades financieras no pueden darles crédito debido a que estas personas no cuentan con las condiciones mínimas necesarias para acceder a este.

Otras personas cierran sus emprendimientos productivos o cambian de actividad por varios factores. Entre los más importantes están la falta de claridad en los objetivos y el mercado meta que atienden, ocasionando que no puedan definir una estrategia que sea sostenible en el tiempo. Esta falta de claridad provoca que los(as) microempresarios(as) comiencen a desviarse de la actividad que realizan y a hacer pruebas con productos en los que no son competitivos o con clientes nuevos, de quienes no tienen información sobre sus gustos y preferencias.

Otros elementos que llevan a las empresas a cambiar o cerrar la actividad son exógenos o externos a la dinámica de la empresa. Dentro de ellos se pueden encontrar: enfermedades de 
los(as) empresarios(as) y sus familiares, efectos climáticos adversos, aumento en los gastos del hogar y descapitalización de la empresa.

También existen condiciones en el entorno que limitan su crecimiento; principalmente referidas a la capacidad adquisitiva de las personas, el desarrollo productivo y la calidad de las instituciones. En este último aspecto cabe destacar una serie de cuestionamientos que realizan los(as) microempresarios(as) y que se pueden resumir en lo siguiente:

En el tema financiero, los(as) empresarios(as) se quejan del escaso acceso al sistema financiero formal como mecanismo para acceder al crédito. En el ámbito de la tecnología y la información, lo más destacable es la ausencia de tecnologías adecuadas a las empresas y la escasez de información relevante sobre mercados, oferta, demanda $y$ otros aspectos. Asimismo, en lo que toca a la comercialización, los problemas vienen de la asimetría en la relación entre pequeñas y grandes empresas. Esto último se refleja también en la estructura regulatoria, así como en la presencia de prácticas clientelísticas y corrupción en muchas de las gestiones ante la autoridad (Zevallos, 2003: 61).

\section{EL MÉTODO DE INTERVENCIÓN EN FORMACIÓN EMPRESARIAL}

\subsection{REFERENTE TEÓRICO METODOLÓGICO DE LA} FORMACIÓN EMPRESARIAL

El proyecto FOcCo-MIPYME de la Escuela de Economía de la Universidad Nacional de Costa Rica ofrece una opción de apoyo a las micro, pequeñas y medianas empresas del país por medio de procesos de formación empresarial e investigaciones sectoriales. El principal compromiso asumido por el proyecto es con los sectores productivos y sociales más necesitados, ya que la empresariedad ha resultado ser un mecanismo eficiente para el mejoramiento de las condiciones socioeconómicas de las personas en condiciones de pobreza y vulnerabilidad social.

La ilustración 1 muestra el marco lógico y los mecanismos de intervención que el proyecto ha seguido para realizar los procesos en formación empresarial, los cuales son de extensión, pero poseen un alto componente de investigación e incorporan la docencia; es decir, es un mecanismo de intervención integral brindado desde la universidad a $\operatorname{los}(\mathrm{as})$ microempresarios(as) para mejorar el desarrollo socioeconómico de las personas que participan en ellos. 
MEJORAR LAS

CAPACIDADES COMPETITIVAS MIPYMES

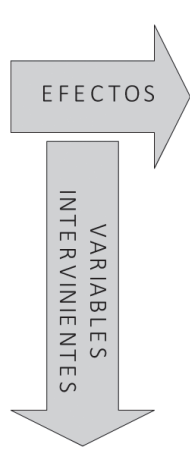

¿Cuáles son las CONDICIONES SOCIO-ECONÓMICAS de los (as) EMPRESARIOS (AS)?

¿Cuáles son los FACTORES CRÍTICOS que condicionan la competitividad de las MIPYMES?

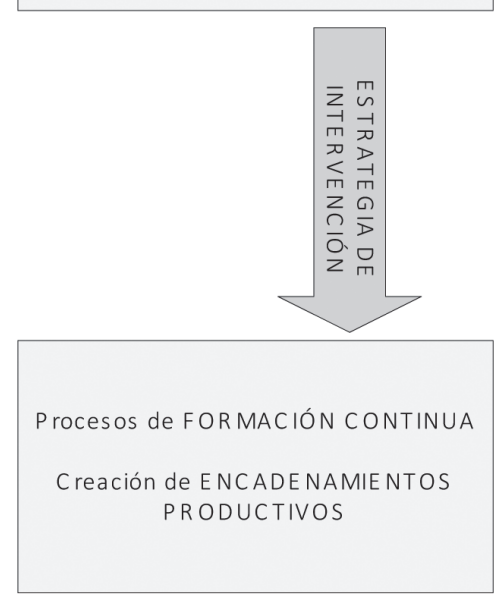

\section{BIENESTAR ECONÓMICO}

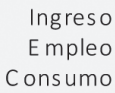

Consumo

Miembros familiares beneficiados Vinculación con otras actividades productivas Aumento en las ventas Solución de necesidades Mejoramiento en el proceso productivo Participación en Actividades Económicas No tradicionales

\section{BIENESTAR SOCIAL}

Alimentación, vestimenta, salud, medicamentos, otros. S ervicios básicos: electricidad, agua, telefonía, otros. Educación

Formación técnica y empresarial

Ampliación en las oportunidades de educación

Mejoras en la vivienda

Vínculo con instituciones

Participación Política

Integración a la sociedad/comunidad

Participación en Actividades sociales no tradicionales

Propiedad de los bienes familiares

\section{BIENESTAR PERSONAL}

Autoes tima Motivación

Dedicación a actividades de Ocio y Personales Adquisición de Bienes Personales Mejoramiento en el aprendizaje Superación personal Ampliación de derechos Seguridad Toma de decisiones Responsabilidad Personal Cambio de liderazgo en el gas to familiar Relaciones de Poder y Toma de Decisiones Reasignación de Tareas Familiares (Productivas-R eproductivas-Ocio)

\section{ILUSTRACIÓN 1}

MECANISMOS DE INTERVENCIÓN EN LOS PROCESOS DE EMPRESARIEDAD

ASUMIDOS POR EL PROYECTO FOCCO-MIPYME

Fuente: Proyecto FOCCO-MYPIME. Escuela de Economía. Universidad Nacional de Costa Rica. 
El objetivo del proyecto FOCCO-MIPYME es desarrollar las capacidades competitivas de las personas dueñas de las micro, pequeñas y medianas empresas costarricenses para enfrentar la competición en el mercado local, nacional e internacional. Estas mejoras en las unidades productivas generarán efectos en el bienestar socioeconómico de las personas que integran las empresas, los cuales pueden ser identificados en tres niveles: económico, social $y$ personal. El vector económico del bienestar está determinado por la capacidad de la empresa para potenciar la producción, los ingresos $y$ el consumo de los hogares. El componente social corresponde a la forma en que las personas participan y se integran a la sociedad y las condiciones en que lo hacen (vivienda, educación, servicios básicos y salud). A nivel personal, cabe esperar que una mayor competitividad de la empresa permita mejorar los niveles de autoestima, motivación y realización personal. En la ilustración 1, se detalla las principales variables que intervienen a cada nivel en el bienestar socioeconómico de las personas.

Sin embargo, el vínculo entre el mejoramiento de las capacidades competitivas de las MiPyMEs y el avance en el bienestar socioeconómico de las personas está condicionado por la situación socioeconómica inicial de los(as) empresarios(as), los factores críticos que afectan la competitividad de las unidades productivas y la estrategia de intervención. Los mayores niveles de productividad, eficiencia, eficacia, producción e ingresos de las empresas se obtienen mediante procesos de intervención en formación empresarial y generación de encadenamientos, los cuales deben considerar, para su formulación y ejecución, las condiciones socioeconómicas de las personas que participarán y los elementos que están limitando la competitividad de estos negocios.

Es sólo a través de una evaluación de las necesidades requeridas por los(as) microempresarios(as) que es posible desarrollar un proceso de capacitación y de seguimiento que logre el mejoramiento continuo a lo largo del ciclo de vida de los proyectos productivos, brindándoles sostenibilidad. La estrategia de intervención utilizada por el proyecto para mejorar la formación empresarial parte de un proceso de investigación que trata de responder a las interrogantes: ¿cuáles son los factores críticos que condicionan la competitividad de las MiPyMEs? y ¿cuáles son las condiciones socioeconómicas de los(as) empresarios(as)?

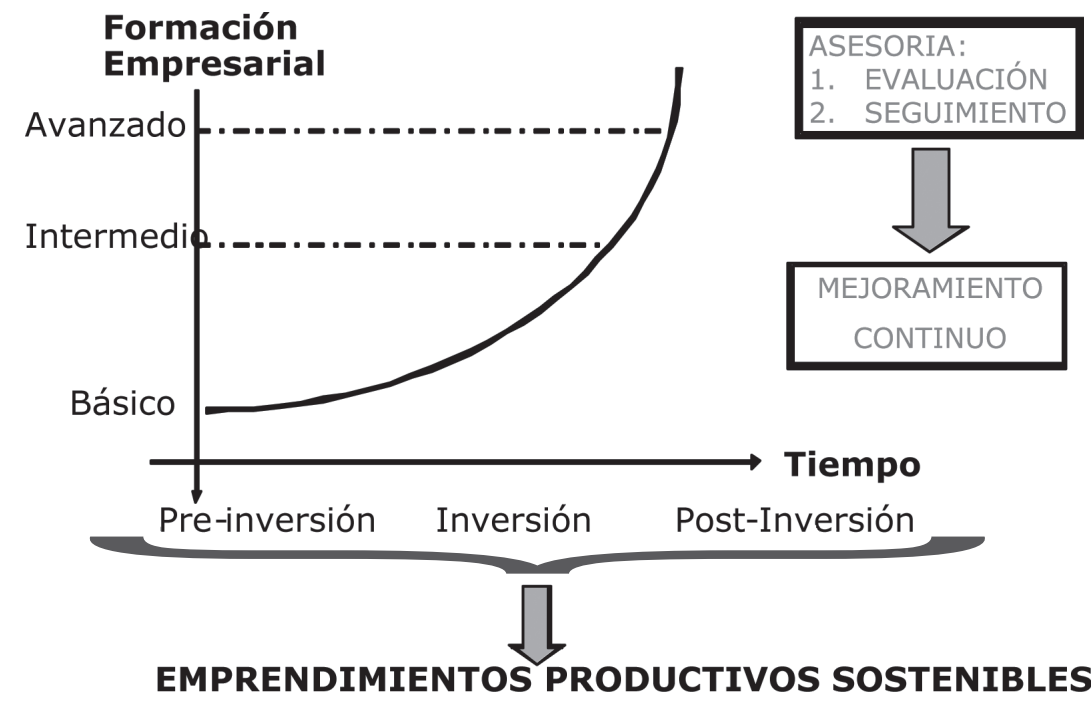

\footnotetext{
ILUSTRACIÓN 2

FORMACIÓN EMPRESARIAL CONTINUA DEL PROYECTO FOCCO-MIPYME

Fuente: Proyecto FOCCO-MIPYME. Escuela de Economía. Universidad Nacional de Costa Rica.
} 
El ciclo de vida de un proyecto productivo sostenible atraviesa tres etapas: pre-inversión, inversión y post-inversión; en las cuales demanda mayores niveles de formación empresarial según trascurre el tiempo. A su vez, las áreas estratégicas en las que se debe enfocar el mejoramiento de la formación empresarial también varían de acuerdo con la etapa en la que se ubique la empresa. Una estrategia de intervención que procure aumentar las capacidades competitivas de las MiPyMEs debe considerar estas características para brindarles una mejor solución.

Los emprendimientos o ideas productivas se ubican en la etapa de pre-inversión y el nivel de formación empresarial que presenta es básico o nulo. Las personas que emprenden en una actividad productiva lo hacen porque identificaron una oportunidad de negocio $y$ tienen experiencia, capacidad y habilidad técnica para producir o comercializar, aunque no posean un nivel de conocimiento adecuado sobre la gestión de la empresa. Con estas condiciones de formación empresarial el proyecto no tiene las bases para llevar a cabo mejoras que le permitan ser más productivo y eficiente, condenándose a niveles de ingreso de subsistencia o a una vida muy corta. Por lo tanto, la capacitación en áreas estratégicas de la gestión de empresas se hace necesaria desde la etapa inicial de la idea productiva.

En la etapa de inversión, los(as) empresarios(as) deberán tener capacidad para decidir sobre la adecuada distribución de los recursos disponibles, conocer los requisitos para iniciar operaciones $y$ tener claro como organizará la gestión de la empresa.

Durante la etapa de post-inversión del proyecto, la formación empresarial debe asumirse de forma continua para que el(la) empresario(a) pueda adaptarse a los cambios del entorno y realizar procesos productivos eficientes $y$ de calidad. En esta etapa, los(as) empresarios(as) deberán tener un nivel adecuado para administrar las operaciones de la empresa, tomar decisiones sobre nuevas inver- siones y definir la orientación a largo plazo. Para ser competitivos a nivel nacional e internacional, los(as) empresarios(as) deberán ampliar las operaciones e innovar los productos $y$ procesos que realizan en las empresas; $y$ para ello requerirán mejorar su nivel de conocimiento y recibir asesoría.

Lo anterior, conllevará a la consecución de mejores niveles de empleo, producción e ingresos, avanzándose en el mejoramiento del nivel de vida en las regiones en las que se desarrollen dichos proyectos.

\section{METODOLOGÍA DE INTERVENCIÓN EN FORMACIÓN EMPRESARIAL}

La filosofía de trabajo del proyecto se fundamenta en la necesidad de un mejoramiento continuo en las capacidades competitivas de los sectores productivos del país, con el fin de obtener el mayor efecto posible sobre el nivel de formación empresarial de los(as) microempresarios(as). La estrategia de intervención del proyecto FOCCO-MIPYME en formación empresarial se realiza mediante procesos de educación continua impartidos en talleres, seminarios y cursos de capacitación en empresariedad, las cuales se complementan con procesos de investigación, evaluación, seguimiento y asesoría.

El proceso inicia con un diagnóstico de las condiciones socioeconómicas y las necesidades en materia de empresariedad de los(as) empresarios(as), a través de la construcción, la aplicación y el procesamiento de un instrumento que recolecte esta información.

La segunda etapa de intervención consiste en la realización de talleres de capacitación en áreas estratégicas de gestión empresarial de acuerdo con las necesidades de aprendizaje de cada uno de los grupos, así, se puede profundizar en temas como estructura de costos, mercadeo y comercialización, formalización de empresas, gestión empresarial, entre otros. La siguiente tabla detalla los módulos que se han desarrollado más comúnmente por parte del proyecto FOCCO-MIPYME. 
TABLA 1

PRINCIPALES MÓDULOS DE CAPACITACIÓN

IMPARTIDO POR EL PROYECTO FOCCO-MIPYME

SEGÚN ETAPA EN EL CICLO DE INVERSIÓN DE LA EMPRESA

\begin{tabular}{|c|}
\hline Pre-Inversión \\
\hline $\begin{array}{l}\diamond \text { Autoestima y Liderazgo } \\
\diamond \text { Organización y Liderazgo } \\
\diamond \text { Formulación de } \\
\text { Proyectos } \\
\diamond \text { Gestión Estratégica } \\
\diamond \text { Emprendedurismo } \\
\diamond \text { Tramitología para } \\
\text { Mipyme } \\
\diamond \text { Planes de Negocios }\end{array}$ \\
\hline
\end{tabular}
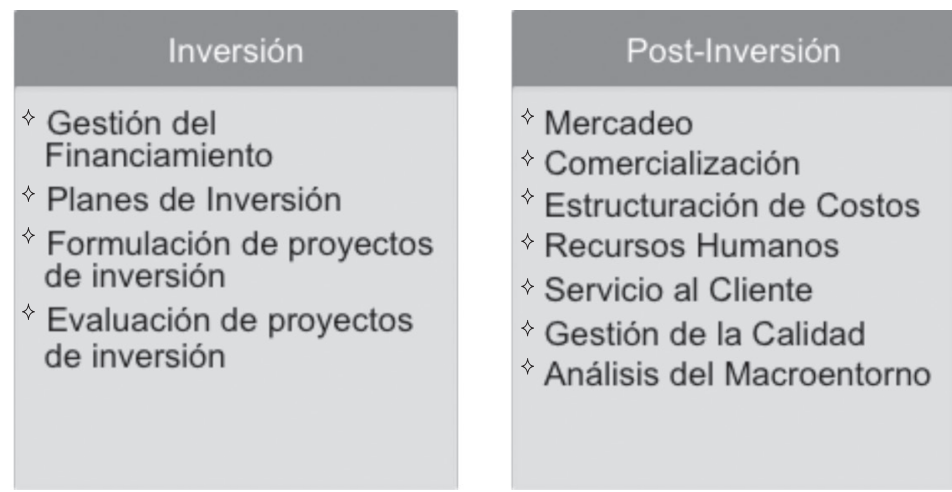

Fuente: Proyecto FOcCO-MYPIME. Escuela de Economía. Universidad Nacional de Costa Rica.

La tercera fase de la intervención es un proceso de seguimiento de los emprendimientos productivos. Las áreas que profundiza este proceso están definidas por los cursos de capacitación que los(as) microempresarios(as) culminaron en la etapa anterior del programa, los cuales fueron determinados a partir del diagnóstico de empresariedad. La estrategia metodológica consiste en:

$\diamond \quad$ Talleres, en los cuales se repase y retomen los elementos teóricos necesarios para avanzar en el seguimiento.

$\diamond \quad$ Visitas a los(as) microempresarios(as), donde el capacitador tiene un acercamiento a las actividades productivas de cada uno de los(as) microempresarios(as) $y$ vela por la correcta aplicación del conocimiento adquirido.

$\diamond \quad$ Bitácora, donde se registre todo el proceso de seguimiento.

$\diamond \quad$ Productos finales de los microempresarios, los cuales consisten en planes de marketing, formulaciones de proyectos $y$ documentos de gestión estratégica, según se haya definido previamente en un "contrato de competitividad". El seguimiento permite medir o evaluar el impacto generado por parte del proceso de capacitación en el mejoramiento de las capacidades competitivas de las microempresas atendidas.

El proceso de seguimiento concluye con la elaboración de los productos que se detallen en la estrategia metodológica, principalmente: planes de negocios, elaboración de un proyecto, elaboración de una estrategia de estructuración de costos, planes de comercialización y mercadeo, entre otras.

Durante todo el proceso se identifican lineamientos estratégicos y de política pública, con el fin de realizar recomendaciones que fomenten el mejoramiento continuo de las capacidades competitivas de los emprendimientos productivos y microempresas del cantón atendido.

El proceso de seguimiento permite a los(as) investigadores(as) compartir con los(as) microempresarios(as) y así diagnosticar aquellos factores que se requieren para aumentar los niveles de eficacia y eficiencia, a la vez que se buscan soluciones reales a las necesidades de estos. Por consiguiente, las recomendaciones de lineamientos estratégicos y políticas públicas para el mejoramiento de las capacidades competitivas de estos sectores productivos, se hacen mediante la investigación y la interacción directa con el objeto de trabajo, las microempresas y su entorno. 


\section{RESULTADOS DE LA INTERVENCIÓN EN FORMACIÓN EMPRESARIAL}

Los resultados del proyecto han evidenciado mejoras en el nivel económico, social y personal de los(as) participantes en los procesos de capacitación y seguimiento. Formar parte de los procesos de formación continua ha generado que los(as) microempresarios(as) puedan contar con mayores herramientas que hagan más competitiva su empresa.

Al realizar dinámicas participativas en las sesiones de trabajo, los(as) empresarios(as) han mejorado los grados de motivación, autoestima y superación personal.

Por otro lado, el proyecto ha podido identificar claramente las necesidades en formación empresarial que tienen los(as) empresarios(as) a través de investigación en temas como organización, desempeño y factores críticos de la competitividad de los sectores productivos.

La metodología utilizada ha sido bien aceptada por las instituciones y los(as) empresarios(as) con que ha trabajado el proyecto. Diagnosticar las condiciones socioeconómicas de los(as) empresarios(as) y poseer una estrategia de formación empresarial de carácter continuo, conformada por procesos de evaluación, capacitación, asesoría y seguimiento, la cual considere las necesidades de formación empresarial en las distintas etapas del proyecto, son ventajas distintivas muy valoradas que se han alcanzado. A su vez, esto permite que el desarrollo del proceso de formación empresarial se adapte a las necesidades reales de las personas e instituciones.

El proyecto ha creado alianzas muy importantes con otras instituciones y proyectos que fortalecen el quehacer y mejoran los resultados obtenidos en la extensión. La necesidad de mejorar las capacidades competitivas de la MiPyMEs es una labor que requiere de diferentes soluciones; por lo que, es necesaria la participación de diferentes actores.

En la experiencia del proyecto existen muchos casos exitosos cuyas mejoras son de diversa índole. Para mostrar esta diversidad de canales de transmisión de las mejoras de la formación empresarial, se expondrán tres de estos casos. La recopilación de la información que aquí se presenta se hizo mediante las bitácoras elaboradas en procesos de seguimiento empresarial que el proyecto realizó.

El primer caso corresponde a Marisel Zúñiga Leiva, empresaria de Buenos Aires de Puntarenas. Esta empresaria inició con una venta de pañales en la estación de buses de Buenos Aires con un nivel de ventas de $\$ 10 \quad 000,00$ diarios. Como beneficiaria del proyecto recibió capacitación, seguimiento $y$ asesoría en mercadeo $y$ estructuración de costos. En el área de mercadeo, se trabajó sobre la ubicación más optima para un posible local comercial, la variedad de productos a ofrecer en la tienda, la promoción y la presentación de puntos de ventas; por otro lado, la parte de estructuración de costos se enfocó al registro de las actividades, control de los inventarios y fijación de precios de productos comerciables.

Los resultados obtenidos gracias a la mejora en formación empresarial, se evidencian en la apertura de otra tienda en la estación de buses de San Isidro del General, además, incrementó la variedad de productos, adquirió un rótulo publicitario, mejoró su control y registro de inventarios y ventas diarias detalladas, así como lograr contratar tres trabajadoras. Estas mejoras significativas han hecho que el local de Buenos Aires tenga niveles de venta de $\$ 40000,00$ diarios y el de San Isidro del General $\$ 450$ 000,00 diarios. Estos beneficios se han trasladado al bienestar socioeconómico de ella y sus hijas, además, de apoyar a su hermana, pues le ha cedido la mitad del negocio de Buenos Aires para que ella se encargue y trate de progresar.

Otro caso exitoso es el de Obed Gamboa Cerdas y su empresa "Solo Cuero". Este empresario ha recibido bastante apoyo institucional, pues el Instituto Mixto de Ayuda Social (IMAS), la Universidad Nacional de Costa Rica (UNA), el Instituto Nacional de Aprendizaje (INA), el Banco Popular, el Banco Nacional de Costa Rica y la Promotora de Comercio Exterior (PROCOMER) le han brindado diferentes formas de ayuda, permitiéndole mejorar sus capacidades competitivas. En la parte técnica, está muy bien preparado gracias a los cursos que ha tomado en el INA, puesto que nadie en la 
zona elabora los productos de cuero (fajas $y$ bolsos) con tan alta calidad como él lo hace. Por parte de los bancos y el IMAs, ha recibido financiamiento y promoción por medio de ferias. Dentro del proyecto se benefició con capacitación en estructuración de costos y mercadeo estratégico. En el área de mercadeo estratégico se hizo énfasis en la creación de un catálogo fotográfico para promocionar la oferta de la empresa. En el seguimiento en costos, se trabajó la contabilización de los residuos de cuero y el impacto que ello tiene en la estructura de costos, con base a ello se planteó la estrategia de elaboración de productos a partir de los residuos (productos secundarios), además se trabajó en la fijación de precios, así como, en la programación de la producción y técnicas para mejorarla.

A partir del proceso de capacitación, asesoría y seguimiento, Obed decidió crear moldes para elaborar los cortes del producto y producir fajas para perros, a partir de los residuos de cuero que se producen. También, se asesoró para comprar una máquina prensadora que le permite hacer los cortes en menos tiempo y estandarizados. Todas estas mejoras han ampliado la capacidad competitiva, el nivel de ventas y la incorporación de los miembros de la familia en la empresa. No obstante, Obed posee el gran reto de contratar personal para que le ayude a ampliar su producción a un nivel que le permita exportar, tarea que se ha dificultado debido a la baja cualificación de la mano de obra en la zona.

Otro caso exitoso de los procesos de seguimiento fue el de Ana Lorena Godínez, quien se dedica a confeccionar prendas de vestir, por lo cual participó en un proceso de capacitación, asesoría y seguimiento en gestión estratégica y mercadeo. Con ella se trabajó en la elaboración de materiales publicitarios, definición de mercado meta y la línea de productos a ofrecer. En la parte de gestión estratégica, se precisó la misión de la empresa, así como su visión, la cual se basaba en la necesidad de que la empresa fuera un complemento para el ingreso familiar, sin obstaculizar su trabajo de ama de casa, ni la educación de sus hijos. Para ello se definieron metas de ventas mensuales, que fueran asequibles con la disponibilidad de horario con que contaba la empresaria. Lo particular de este caso, es que el éxito que ella posee obedece a que se logró compatibilizar las labores domésticas y empresariales de acuerdo a los deseos de la empresaria. Se considera que esto fue el impulsor del éxito alcanzado, pues en muchas ocasiones, principalmente en empresas lideradas por mujeres, existe gran rivalidad entre las distintas labores que debe desempeñar la mujer.

\section{CONCLUSIONES}

Se debe destacar que las pequeñas y medianas empresas se enfrentan a una estructura de mercado muy competitiva y dinámica, en donde las economías de escala, la innovación y la participación de empresas trasnacionales hacen cada vez más difícil el mantenerse en el mercado.

Por otra parte, existen limitantes dentro de la capacidad operativa de las empresas, estas han sido claramente evidenciadas en los diagnósticos aplicados dentro del Proyecto FOCCO-MYPIME de la Universidad Nacional y se pueden destacar la falta de planificación estratégica a largo plazo, la ausencia de programas de mercadeo y comercialización, la ausencia de estructuración y registro de costos y ventas, la escasa capacidad para acceder a financiamiento, como algunas de los principales cuellos de botella. Ante este panorama evidentemente pesimista, el Proyecto Focco ha podido evidenciar mediante sus procesos de capacitación y seguimiento en la región Brunca del país que estas capacidades pueden ser creadas, es decir, las personas pueden desarrollar habilidades y procesos que pueden ser replicados en sus microempresas $y$ de esa forma potencializar la competitividad de las mismas, garantizándose un mayor éxito financiero y por ende, una mejor calidad de vida para las familias de $\operatorname{los}($ as) empresarios(as).

Otro de los hallazgos es que los procesos de capacitación y seguimiento pueden solventar los problemas y generar mayores capacidades en las personas, si se realizaran periodos de seguimiento y asesoría más prolongados. 


\section{RECOMENDACIONES DE EXTENSIÓN}

Algunas recomendaciones mínimas que deben de tomarse en cuenta cuando se realizan procesos de capacitación y seguimiento a los microempresarios son:

1. Los capacitadores deben conocer profundamente la población a la cual van a atender, así mismo, se debe conocer el entorno donde se trabajará, variables como nivel de ingreso, pobreza, nivel educativo de la población meta, entre otros aspectos relevantes.

2. El extensionista debe adaptar las estrategias de intervención a las necesidades particulares de cada microempresario(a), sin bien es cierto, los cursos de capacitación pueden ser homogéneos, el seguimiento $y$ asesorías deben adecuarse a cada caso.

3. Los cursos deben ser integrales, es decir, deben incorporar tanto el componente teórico y práctico, así como, procesos de retroalimentación con experiencias de los(as) mismos(as) participantes. Es necesario que los procesos de extensión realizados por las instituciones de educación superior orientados a mejorar las capacidades competitivas de las micro, pequeñas $y$ medianas empresas se realicen bajo esquemas de intervención integrales, debido a la naturaleza de los factores críticos que afectan la sostenibilidad de las microempresas y la capacidad de mejorar el bienestar socioeconómico de personas en condiciones de pobreza.

4. Un cuarto elemento esencial en los procesos (pero que pocas veces se concreta) es que debe realizarse una evaluación del impacto de la intervención realizada, es decir, se debe conocer si los procesos están generando capacidades dentro de las personas que permitan que sus microempresas comiencen a mejorar y ser más exitosas.

5. Finalmente, la formación empresarial debe ser complementada con otro tipo de procesos que satisfagan las necesidades de conocimientos que generalmente presentan los pequeños empresarios. En este sentido, la capacitación técnica, la accesibilidad a vivienda, el mejoramiento del nivel educativo, el financiamiento, entre otros aspectos deben ser resueltos. Se debe tener presente que la formación empresarial constituye un buen soporte para otro tipo de políticas sociales como vivienda, educación, terrenos y fomento de organizaciones como lo son las asociaciones de desarrollo, cooperativas y juntas de educación.

\section{BIBLIOGRAFÍA}

FOCCO MIPYMES-IMAS. Evaluación del efecto del crédito en el bienestar socioeconómico $y$ en la formación empresarial de los(as) beneficiarios(as) apoyados por el IMAS a través del Fideicomiso, Proceso SP-002-2006. Heredia, Costa Rica: Escuela de Economía de la Universidad Nacional, 2007.

FOCCO MIPYMES-IMAS. Evaluación de los efectos de los programas del Fideicomiso (fondos de garantía y servicios de apoyo) en el bienestar socioeconómico y capacidad competitiva de los(as) beneficiarios(as) y sus empresas apoyadas en el periodo 2005-2007. Heredia, Costa Rica: Escuela de Economía de la Universidad Nacional, 2009.

FOCCO MIPYMES-IMAS. "Formación Continua de las Capacidades Competitivas de las Micro, Pequeñas y Medianas Empresas de Costa Rica (FOCCO-MIPYME) COD: 023918". Informe final. Heredia, Costa Rica: Escuela de Economía de la Universidad Nacional de Costa Rica, 2009.

Gómez, M. "Competitividad de las PyMEs: ¿cómo pueden las pequeñas y medianas empresas de Costa Rica competir en el mercado local e internacional?". Revista Economía y Sociedad 19. Universidad Nacional de Costa Rica, 2002. 
Picón, J.C.; Baltodano, V. y Parada, M. "La microempresa y su inserción en el conglomerado empresarial turístico: 11 caso del Golfo de Papagayo". Cuaderno de Trabajo. Costa Rica (Nicoya): Universidad Nacional, Centro Mesoamericano de Desarrollo Sostenible del Trópico Seco. 2006.

Presidencia de la República y Ministerio de Economía, Industria y Comercio de
Costa Rica. Definición de empresa para efectos de Reconversión Productiva. San José. Costa Rica, 1997.

Zevallos, Emilio. "Micro, pequeñas y medianas empresas en América Latina". Revista de la CEPAL 79. Santiago de Chile, 2003.

Fecha de ingreso: 30/01/2012 Fecha de aprobación: 27/04/2012 
